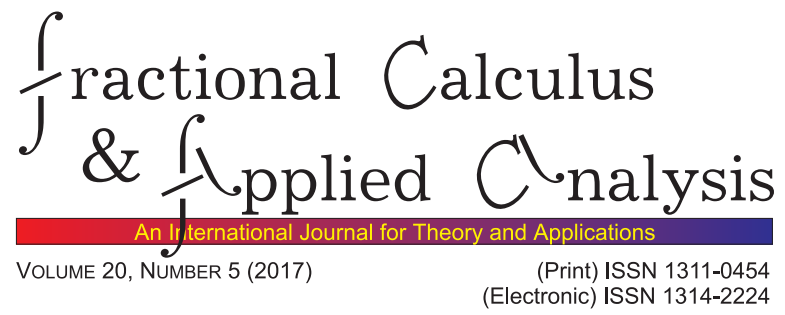

RESEARCH PAPER

\title{
INFINITELY MANY SIGN-CHANGING SOLUTIONS FOR THE BRÉZIS-NIRENBERG PROBLEM INVOLVING THE FRACTIONAL LAPLACIAN
}

\author{
Lin Li ${ }^{1}$, Jijiang Sun ${ }^{2, *}$, Stepan Tersian ${ }^{3,4}$ \\ Dedicated to Professor Virginia Kiryakova on the occasion \\ of her 65th birthday and the 20th anniversary of FCAA
}

\begin{abstract}
In this paper, we consider the following Brézis-Nirenberg problem involving the fractional Laplacian operator:$$
\begin{cases}(-\Delta)^{s} u=\lambda u+|u|^{2_{s}^{*}-2} u & \text { in } \Omega \\ u=0 & \text { on } \partial \Omega\end{cases}
$$

where $s \in(0,1), \Omega$ is a bounded smooth domain of $\mathbb{R}^{N}(N>6 s)$ and $2_{s}^{*}=\frac{2 N}{N-2 s}$ is the critical fractional Sobolev exponent. We show that, for each $\lambda>0$, this problem has infinitely many sign-changing solutions by using a compactness result obtained in 34 and a combination of invariant sets method and Ljusternik-Schnirelman type minimax method.
\end{abstract}

$M S C$ 2010: Primary 35J60; Secondary 47J30, 58E05

Key Words and Phrases: fractional critical exponent, sign-changing solutions, invariant sets, minimax method

\section{Introduction and main results}

In this paper, we consider the following nonlinear problem with the fractional Laplacian

$$
\begin{cases}(-\Delta)^{s} u=\lambda u+|u|^{2_{s}^{*}-2} u & \text { in } \Omega \\ u=0 & \text { on } \partial \Omega\end{cases}
$$

(C) 2017 Diogenes Co., Sofia

pp. 1146-1164, DOI: 10.1515/fca-2017-0061

DE GRUYTER 
INFINITELY MANY SIGN-CHANGING SOLUTIONS FOR . . 1147

where $\lambda>0,0<s<1, \Omega$ is a bounded smooth domain of $\mathbb{R}^{N}$ and $2_{s}^{*}=\frac{2 N}{N-2 s}$ is the critical exponent in fractional Sobolev inequalities. Here the fractional Laplacian $(-\Delta)^{s}$ is defined as follows.

Let $\left\{\varphi_{k}\right\}$ be an orthonormal basis of $L^{2}(\Omega)$ with $\left\|\varphi_{k}\right\|_{L^{2}(\Omega)}=1$ forming a spectral decomposition of $-\Delta$ in $\Omega$ with zero Dirichlet boundary data and $\lambda_{k}$ be the corresponding eigenvalues, i.e. $-\Delta \varphi_{k}=\lambda_{k} \varphi_{k}$ in $\Omega$ with $\varphi_{k}=0$ on $\partial \Omega$. Let $0<s<1$ and

$$
H_{0}^{s}(\Omega)=\left\{u=\sum_{k=1}^{\infty} u_{k} \varphi_{k} \in L^{2}(\Omega):\|u\|_{H_{0}^{s}(\Omega)}=\left(\sum_{k=1}^{\infty} \lambda_{k}^{s} u_{k}^{2}\right)^{1 / 2}<\infty\right\}
$$

be the fractional Sobolev space (see [2, 37]) with inner product

$$
(u, v)_{H_{0}^{s}(\Omega)}=\sum_{0}^{\infty} \lambda_{k}^{s} u_{k} v_{k}=\int_{\Omega}(-\Delta)^{s / 2} u \cdot(-\Delta)^{s / 2} v d x
$$

It is not difficult to see that $H_{0}^{s}(\Omega)$ is a Hilbert space. For any $u \in H_{0}^{s}(\Omega)$, $u=\sum_{k=1}^{\infty} u_{k} \varphi_{k}$ with $u_{k}=\int_{\Omega} u \varphi_{k} \mathrm{~d} x$, the spectral fractional Laplacian $(-\Delta)^{s}$ is defined by

$$
(-\Delta)^{s} u=\sum_{k=1}^{\infty} \lambda_{k}^{s} u_{k} \varphi_{k}
$$

We say that $\left\{\left(\varphi_{k}, \lambda_{k}^{s}\right)\right\}$ are the eigenfunctions and eigenvalues of $(-\Delta)^{s}$ in $\Omega$ with zero Dirichlet boundary data. In the pioneering work [6], Brézis and Nirenberg considered the existence solutions of equation (1.1) with $s=1$. They show that for $\lambda>0$ the problem

$$
\begin{cases}-\Delta u=\lambda u+|u|^{2^{*}-2} u & \text { in } \Omega \\ u>0 & \text { in } \Omega \\ u=0 & \text { on } \partial \Omega\end{cases}
$$

where $2^{*}=\frac{2 N}{N-2}$, may admit non-trivial solutions under a subtle dependence on the dimension $N \geq 3$. After that, there are many results on this problem. See e.g. [14, 15, 17, 28] and the references therein. In particular, in [18], Devillanova and Solimini showed that, when $N \geq 7$, problem (1.1) with $s=1$ has infinitely many solutions for each $\lambda>0$ by using the uniform bounded theorem (see Theorem 1.1 in [18]). Recently, under the same assumptions of [18], Schechter and Zou [23] proved that this problem has infinitely many sign-changing solutions by combining the estimates of Morse indices of nodal solutions with the uniform bounded theorem due to Devillanova and Solimini [18].

Nonlinear problems involving the fractional Laplacian have been extensively studied recently. Caffarelli et al. [8, 9] studied the free boundary problem for the fractional Laplacian. Silvestre [27] investigated the regularity of the obstacle problem for the fractional Laplace operator. In [10], 
Caffarelli and Silvestre given a new local realization of the fractional Laplacian $(-\Delta)^{s}$ by introducing the so-called $s$-harmonic extension. After that, several authors, using the localization method, have extended some results of the classical elliptic problems to the fractional Laplacian, see for example [2, 5, 7, 13, 16, 31, 34, 35, 36, 24, 25, 26] and the references therein. In particular, Chang and Wang [16, using the method of invariant sets of descending flow, obtained the existence and multiplicity of nodal solutions for the elliptic equaitons involving the fractional Laplacian $(-\Delta)^{s}$ for all $s \in(0,1)$ with subcritical nonlinearities; for the Brézis-Nirenberg type problem involving the fractional Laplacian (1.1), Tan [31] proved the existence of positive solutions with the special case $s=\frac{1}{2}$ and Barrios et al. [2] studied the general case with $0<s<1$. For any $\lambda>0$, Yan et al. [34] proved that problem (1.1) possesses infinitely many solutions by using a compactness result for the subcritical perturbed problem associated to (1.1). In 21] the authors study bifurcation and multiplicity of solutions for the fractional Laplacian with critical exponential nonlinearity using critical point theorem of Bartolo, Benci and Fortunato [3. Multiplicity of solutions for fractional differential equations via variational method is studied in [1, 32, 37.

A natural question is whether problem (1.1) has infinitely many signchanging solutions for each $\lambda>0$ and $s \in(0,1)$. To the best of our knowledge, there is no result in the literature concerning this question. In this paper, we give a positive answer to this open question. The main result of this paper is the following.

Theorem 1.1. Suppose that $N>6 s$ and $\lambda>0$, then problem (1.1) has infinitely many sign-changing solutions.

REMark 1.1. Denote $\lambda_{1}^{s}$ the first eigenvalue of $(-\Delta)^{s}$ in $\Omega$ with zero Dirichlet boundary condition. Multiplying the first eigenfunction and integrating both sides, one can easily check that if $\lambda \geq \lambda_{1}^{s}$, any nontrivial solution of (1.1) is sign-changing. Therefore, by the results of [34], to prove Theorem 1.1, it suffices to consider the case of $\lambda \in\left(0, \lambda_{1}^{s}\right)$.

Theorem 1.1 extends the result in 23 to the fractional Laplacian. Motivated by [30] which used the more simple proof than [23] to obtain the same result, we will prove Theorem 1.1 by applying the usual Ljusternik-Schnirelman type minimax method in conjunction with invariant set method. However, due to the fact that the operator $(-\Delta)^{s}$ is nonlocal, the techniques of constructing invariant sets of descending flow in [4, 19, 20] cannot be directly applied to problem (1.1). In order to construct 
INFINITELY MANY SIGN-CHANGING SOLUTIONS FOR . . 1149

invariant sets, we adopt an idea from [13, 16] to introduce an auxiliary operator $A_{\varepsilon}$ (see Section 3) associated to the subcritical perturbed problem (2.3). Then we can follow the same way as in 30] with the help of the compactness result (Theorem 2.1, see Section 24) due to Yan et al. 34] to obtain Theorem 1.1 .

This paper is organized as follows. In Section 2, we describe a variational setting of the problem and state a compactness result due to Yan et al. 34] for the solutions of the perturbed problem (2.4). In Section 3, we introduce an auxiliary operator $A_{\varepsilon}$ and then construct the invariant sets, the proof of Theorem 1.1 is given at the end of this section.

\section{Preliminaries and functional setting}

Denote $H^{-s}(\Omega)$ the dual space of $H_{0}^{s}(\Omega)$. Define the inner product in $H_{0}^{s}(\Omega)$ by

$$
(u, v)_{H_{0}^{s}(\Omega)}:=\int_{\Omega}(-\Delta)^{s / 2} u(-\Delta)^{s / 2} v d x .
$$

Definition 2.1. We say that $u \in H_{0}^{s}(\Omega)$ is a weak solution of (1.1) if the identity

$$
\int_{\Omega}(-\Delta)^{s / 2} u(-\Delta)^{s / 2} \phi d x=\int_{\Omega}\left(\lambda u \phi+|u|^{2_{s}^{*}-2} u \phi\right) d x
$$

holds for every $\phi \in H_{0}^{s}(\Omega)$.

Note that the right hand side of the identity in the above definition is well defined, since $\phi \in H_{0}^{s}(\Omega) \hookrightarrow L^{2_{s}^{*}}(\Omega)$, and for $u \in H_{0}^{s}(\Omega), \lambda u+$ $|u|^{2 *}-2 u \in L^{\frac{2 N}{N+2 s}}(\Omega)$. It is standard (see e.g. [22]) to show that the weak solutions of problem (1.1) correspond to the critical points of the energy functional $I: H_{0}^{s}(\Omega) \rightarrow \mathbb{R}$ given by

$$
I(u)=\frac{1}{2} \int_{\Omega}\left|(-\Delta)^{s / 2} u\right|^{2} d x-\frac{\lambda}{2} \int_{\Omega}|u|^{2} d x-\frac{1}{2_{s}^{*}} \int_{\Omega}|u|^{2_{s}^{*}} d x, \quad \forall u \in H_{0}^{s}(\Omega) .
$$

Clearly, $I \in C^{1}\left(H_{0}^{s}(\Omega), \mathbb{R}\right)$.

Define $\mathbb{R}_{+}^{N+1}=\left\{(x, y): x \in \mathbb{R}^{N}, y>0\right\}$, the upper half space in $\mathbb{R}^{N+1}$. Associate to the bounded domain $\Omega$, we consider the cylinder $\mathcal{C}=$ $\Omega \times(0, \infty) \subset \mathbb{R}_{+}^{N+1}$ and denote its lateral boundary by $\partial_{L} \mathcal{C}=\partial \Omega \times[0, \infty)$.

Note that $(-\Delta)^{s}$ is a nonlocal operator, motivated by the work of Caffarelli and Silvestre [10]. Using the so-called $s$-harmonic extension, several authors have considered an equivalent definition of the operator $(-\Delta)^{s}$ defined through the spectral decomposition as above. Then the nonlocal problems can be transformed into a local problem see e.g. [2, 5, 7, 13, 16, 34, 35. 
For a given $u \in H_{0}^{s}(\Omega)$, we define its $s$-harmonic extension $w=E_{s}(u)$ to $\mathcal{C}$ as the solution of the problem

$$
\begin{cases}-\operatorname{div}\left(y^{1-2 s} \nabla w\right)=0 & \text { in } \mathcal{C} \\ w=0 & \text { on } \partial_{L} \mathcal{C}, \\ w(x, 0)=u & \text { on } \Omega\end{cases}
$$

Following [10], we can define the fractional Laplacian operator by the Dirichlet to Neumann map as follows.

Definition 2.2. For any $u \in H_{0}^{s}(\Omega)$, the fractional Laplacian $(-\Delta)^{s}$ acting on $u$ is defined by

$$
(-\Delta)^{s} u(x):=-\frac{1}{k_{s}} \lim _{y \rightarrow 0^{+}} y^{1-2 s} \frac{\partial w}{\partial y} w(x, y), \quad \forall x \in \Omega,
$$

where $w=E_{s}(u)$ and $k_{s}=\frac{2^{1-2 s} \Gamma(1-s)}{\Gamma(s)}$ is a normalization constant.

Define $H_{0, L}^{s}(\mathcal{C})$ as the closure of $C_{0}^{\infty}(\mathcal{C})$ under the norm

$$
\|w\|_{H_{0, L}^{s}(\mathcal{C})}=\left(k_{s} \int_{\mathcal{C}} y^{1-2 s}|\nabla w|^{2} d x d y\right)^{1 / 2} .
$$

Denote by $\operatorname{tr}_{\Omega}$ the trace operator on $\Omega \times\{0\}$ for functions in $H_{0, L}^{s}(\mathcal{C})$ :

$$
\operatorname{tr}_{\Omega} w=w(\cdot, 0), \quad \text { for } w \in H_{0, L}^{s}(C) .
$$

Then for any $w \in H_{0, L}^{s}(C)$, the following trace inequality holds

$$
\left\|t r_{\Omega} w\right\|_{H_{0}^{s}(\Omega)} \leq\|w\|_{H_{0, L}^{s}(\mathcal{C})} .
$$

Moreover, we have the following result (see [2, 16]).

Lemma 2.1 (Lemma 2.3, [16]). (i) $E_{s}(\cdot)$ is an isometry between $H_{0}^{s}(\Omega)$ and $H_{0, L}^{s}(\mathcal{C})$, that is

$$
\|u\|_{H_{0}^{s}(\Omega)}=\left\|E_{s}(u)\right\|_{H_{0, L}^{s}(\mathcal{C})} ;
$$

(ii) For any $w \in H_{0, L}^{s}(\mathcal{C})$, there exists a constant $C$ independent of $w$ such that

$$
\left\|\operatorname{tr}_{\Omega} w\right\|_{L^{r}(\Omega)} \leq C\|w\|_{H_{0, L}^{s}(\mathcal{C})}
$$

holds for all $r \in\left[2,2_{s}^{*}\right]$. Moreover, $H_{0, L}^{s}(\mathcal{C})$ is compactly embedded into $L^{r}(\Omega)$ for every $r \in\left[2,2_{s}^{*}\right)$.

Set

$$
\partial_{\nu}^{s} w(x):=-\frac{1}{k_{s}} \lim _{y \rightarrow 0^{+}} y^{1-2 s} \frac{\partial w}{\partial y} w(x, y) .
$$


INFINITELY MANY SIGN-CHANGING SOLUTIONS FOR . . 1151

With the above extension, from [10], we can transform the nonlocal problem (1.1) into the following local problem

$$
\begin{cases}-\operatorname{div}\left(y^{1-2 s} \nabla w\right)=0 & \text { in } \mathcal{C} \\ w=0 & \text { on } \partial_{L} \mathcal{C} \\ \partial_{\nu}^{s} w=\lambda w(x, 0)+|w(x, 0)|^{2_{s}^{*}-2} w(x, 0) & \text { on } \Omega\end{cases}
$$

A weak solution to this problem is a function $w \in H_{0, L}^{s}(\mathcal{C})$ such that

$$
k_{s} \int_{\mathcal{C}} y^{1-2 s}(\nabla w, \nabla \psi) d x d y=\int_{\Omega}\left(\lambda w(x, 0)+|w(x, 0)|^{2_{s}^{*}} w(x, 0)\right) \operatorname{tr}_{\Omega} \psi d x
$$

for all $\psi \in H_{0, L}^{s}(\mathcal{C})$. Then, critical points of the functional

$$
J(w)=\frac{k_{s}}{2} \int_{\mathcal{C}} y^{1-2 s}|\nabla w|^{2} d x d y-\frac{\lambda}{2} \int_{\Omega}|w(x, 0)|^{2} d x-\frac{1}{2_{s}^{*}} \int_{\Omega}|w(x, 0)|^{2_{s}^{*}} d x,
$$

defined on $H_{0, L}^{s}(\mathcal{C})$ correspond to the solutions of (2.2). For any weak solution $w \in H_{0, L}^{s}(\mathcal{C})$ to (2.2) , the function $u=\operatorname{tr}_{\Omega} w \in H_{0}^{s}(\Omega)$ is a weak solution of problem (1.1) and is a critical point of $I$. The converse is also true. Therefore, these two formulations are equivalent, and we will use both formulations in the sequel.

Given $\varepsilon>0$ small enough, associated to problems (1.1) and (2.2), we consider the following subcritical perturbed nonlocal problem:

$$
\begin{cases}(-\Delta)^{s} u=\lambda u+|u|^{2_{s}^{*}-2-\varepsilon} u & \text { in } \Omega \\ u=0 & \text { on } \partial \Omega\end{cases}
$$

and the local problem

$$
\begin{cases}-\operatorname{div}\left(y^{1-2 s} \nabla w\right)=0 & \text { in } \mathcal{C} \\ w=0 & \text { on } \partial_{L} \mathcal{C} \\ \partial_{\nu}^{s} w=\lambda w(x, 0)+|w(x, 0)|^{2_{s}^{*}-2-\varepsilon} w(x, 0) & \text { on } \Omega\end{cases}
$$

The functional $I_{\varepsilon}: H_{0}^{s}(\Omega) \rightarrow \mathbb{R}$ corresponding to (2.3) is defined as follows $I_{\varepsilon}(u)=\frac{1}{2} \int_{\Omega}\left|(-\Delta)^{s / 2} u\right|^{2} d x-\frac{\lambda}{2} \int_{\Omega}|u|^{2} d x-\frac{1}{2_{s}^{*}-\varepsilon} \int_{\Omega}|u|^{2_{s}^{*}-\varepsilon} d x, \forall u \in H_{0}^{s}(\Omega)$.

It is easy to check that $I_{\varepsilon} \in C^{1}\left(H_{0}^{s}(\Omega), \mathbb{R}\right)$.

Now we state the following compactness result due to Yan et al. [34, which plays an important role in our proof.

Theorem 2.1 ([34], Theorem 1.1). Suppose $N>6 s$ and $\lambda>0$. Assume that $w_{n}(n=1,2, \cdots)$ is a nontrivial solution of (2.4) with $\varepsilon=\varepsilon_{n}>$ 0 , and $\left\{w_{n}\right\}_{n \in \mathbb{N}}$ satisfies $\left\|w_{n}\right\| \leq C$ for some positive constant independent of $n$. Then $\left\{w_{n}\right\}_{n \in \mathbb{N}}$ possesses a subsequence which converges strongly in $H_{0, L}^{s}(\Omega)$ as $n \rightarrow \infty$. 


\section{Proof of the main result}

In this section, we will prove Theorem 1.1.

3.1. Some technical lemmas. Let

$$
0<\lambda_{1}^{s}<\lambda_{2}^{s} \leq \lambda_{3}^{s} \leq \cdots \leq \lambda_{m}^{s} \leq \cdots
$$

be the eigenvalues of $\left((-\Delta)^{s}, H_{0}^{s}(\Omega)\right)$ introduced in Section 1 and $\varphi_{m}$ be the eigenfunction corresponding to $\lambda_{m}^{s}$. Denote

$$
E_{m}:=\operatorname{span}\left\{\varphi_{1}, \varphi_{2}, \cdots, \varphi_{m}\right\} .
$$

Fix $\zeta \in\left(2,2_{s}^{*}\right)$. In the following, we will always assume that

$$
\lambda \in\left(0, \lambda_{1}^{s}\right) \text { and } \varepsilon \in\left(0,2_{s}^{*}-\zeta\right) .
$$

In order to construct the minimax values for the perturbed functional $I_{\varepsilon}$, the following three technical lemmas are needed.

Lemma 3.1. For any $\varepsilon \in\left(0,2_{s}^{*}-\zeta\right)$, the functional $I_{\varepsilon}$ satisfies the Palais-Smale ((PS) for short) condition.

P r o o f. Suppose $\left\{u_{n}\right\} \subset H_{0}^{s}(\Omega)$ is a (PS) sequence for $I_{\varepsilon}$, i.e.,

$$
I_{\varepsilon}\left(u_{n}\right) \rightarrow c \in \mathbb{R} \quad \text { and } \quad I_{\varepsilon}^{\prime}\left(u_{n}\right) \rightarrow 0 \quad \text { as } n \rightarrow \infty .
$$

We have

$$
\begin{aligned}
c+o(1)\left(1+\left\|u_{n}\right\|_{H_{0}^{s}(\Omega)}\right) & =I_{\varepsilon}\left(u_{n}\right)-\frac{1}{2_{s}^{*}}\left\langle I_{\varepsilon}^{\prime}\left(u_{n}\right), u_{n}\right\rangle_{H^{-s}(\Omega), H_{0}^{s}(\Omega)} \\
& =\left(\frac{1}{2}-\frac{1}{2_{s}^{*}}\right)\left(\left\|u_{n}\right\|_{H_{0}^{s}(\Omega)}^{2}-\lambda\left\|u_{n}\right\|_{2}^{2}\right) \\
& \geq\left(\frac{1}{2}-\frac{1}{2_{s}^{*}}\right) \frac{\lambda_{1}^{s}-\lambda}{\lambda_{1}^{s}}\left\|u_{n}\right\|_{H_{0}^{s}(\Omega)}^{2},
\end{aligned}
$$

which implies that $\left\{u_{n}\right\}$ is bounded sequence in $H_{0}^{s}(\Omega)$. Hence, there exists $u_{0} \in H_{0}^{s}(\Omega)$ such that

$$
u_{n} \rightarrow u_{0} \text { in } H_{0}^{s}(\Omega) \text {. }
$$

By Lemma 2.1, we have

$$
u_{n} \rightarrow u_{0} \text { in } L^{r}(\Omega) \text { with } r \in\left[2,2_{s}^{*}\right)
$$

and

$$
u_{n}(x) \rightarrow u_{0}(x) \text { for a.e. } x \in \Omega \text {. }
$$


INFINITELY MANY SIGN-CHANGING SOLUTIONS FOR . . 1153

It follows that

$$
\begin{aligned}
& o(1)=\left\langle I_{\varepsilon}^{\prime}\left(u_{n}\right), u_{n}-u_{0}\right\rangle_{H^{-s}(\Omega), H_{0}^{s}(\Omega)} \\
& =\int_{\Omega}(-\Delta)^{s / 2} u_{n}(-\Delta)^{s / 2}\left(u_{n}-u_{0}\right) d x+\int_{\Omega}\left(\lambda+\left|u_{n}\right|^{2_{s}^{*}-2-\varepsilon}\right) u_{n}\left(u_{n}-u_{0}\right) d x \\
& =\left\|u_{n}-u_{0}\right\|_{H_{0}^{s}(\Omega)}^{2}+o(1), \quad \text { as } n \rightarrow \infty,
\end{aligned}
$$

which implies that

$$
u_{n} \rightarrow u_{0} \text { in } H_{0}^{s}(\Omega)
$$

and the proof is completed.

Lemma 3.2. Suppose $m \geq 1$. Then there exists $R=R\left(E_{m}\right)>0$, such that for all $\varepsilon \in\left(0,2_{s}^{*}-\zeta\right)$,

$$
\sup _{\mathcal{B}_{R}^{c} \cap E_{m}} I_{\varepsilon}<0,
$$

where $\mathcal{B}_{R}^{c}:=H_{0}^{s}(\Omega) \backslash \mathcal{B}_{R}$ and $\mathcal{B}_{R}=\left\{u \in H_{0}^{s}(\Omega):\|u\|_{H_{0}^{s}(\Omega)} \leq R\right\}$.

P r o o f. Define an auxiliary functional $I_{*}: H_{0}^{s}(\Omega) \rightarrow \mathbb{R}$ given by

$$
I_{*}(u)=\frac{1}{2} \int_{\Omega}\left(\left|(-\Delta)^{s / 2} u\right|^{2}-\lambda u^{2}\right) d x-\frac{1}{2_{s}^{*}} \int_{\Omega}|u|^{\zeta} d x .
$$

Noting that

$$
\frac{1}{2_{s}^{*}}\left(|u|^{\zeta}-1\right) \leq \frac{1}{2_{s}^{*}-\varepsilon}|u|^{2_{s}^{*}-\varepsilon},
$$

it is easy to check that

$$
I_{\varepsilon}(u) \leq I_{*}(u)+\frac{|\Omega|}{2_{s}^{*}},
$$

holds for any $\varepsilon \in\left(0,2_{s}^{*}-\zeta\right)$. Since any norm in finite dimensional space is equivalent,

$$
\lim _{\|u\|_{H_{0}^{s}(\Omega)} \rightarrow \infty, u \in E_{m}} I_{*}(u)=-\infty
$$

for any fixed $m \geq 1$. Thus the result follows.

Lemma 3.3. For any $\varepsilon \in\left(0,2_{s}^{*}-\zeta\right)$, there exist $\rho_{\varepsilon}>0$ and $\alpha_{\varepsilon}>0$ such that

$$
\inf _{\partial \mathcal{B}_{\rho_{\varepsilon}}} I_{\varepsilon} \geq \alpha_{\varepsilon}
$$

where $\mathcal{B}_{\rho_{\varepsilon}}=\left\{u \in H_{0}^{s}(\Omega):\|u\|_{H_{0}^{s}(\Omega)} \leq \rho_{\varepsilon}\right\}$. that

P r o o f. For $u \in H_{0}^{s}(\Omega)$, by Lemma 2.1, there exists $C(\varepsilon)>0$ such 


$$
\begin{aligned}
I_{\varepsilon}(u) & =\frac{1}{2}\|u\|_{H_{0}^{s}(\Omega)}^{2}-\frac{\lambda}{2}\|u\|_{2}^{2}-\frac{1}{2_{s}^{*}-\varepsilon}\|u\|_{2_{s}^{*}-\varepsilon}^{2_{*}^{*}-\varepsilon} \\
& \geq \frac{\lambda_{1}^{s}-\lambda}{2 \lambda_{1}^{s}}\|u\|_{H_{0}^{s}(\Omega)}^{2}-\frac{C(\varepsilon)}{2_{s}^{*}-\varepsilon}\|u\|_{H_{0}^{s}(\Omega)}^{2_{s}^{*}-\varepsilon} .
\end{aligned}
$$

Noting that $2<2_{s}^{*}-\varepsilon$, we conclude that there exist $\rho_{\varepsilon}>0$ and $\alpha_{\varepsilon}>0$ such that

as required.

$$
\inf _{\partial \mathcal{B}_{\rho_{\varepsilon}}} I_{\varepsilon} \geq \alpha_{\varepsilon}
$$

3.2. Properties of the operator $A_{\varepsilon}$. In order to introduce an auxiliary operator $A_{\varepsilon}$, which will be used to construct invariants set, we first define the inverse of the operator $(-\Delta)^{s}$ (see [16], Section 4).

Definition 3.1. Given $g \in H^{-s}(\Omega)$, define the operator $T_{s}: H^{-s}(\Omega) \rightarrow$ $H_{0}^{s}(\Omega)$, by $T_{s}(g)=\operatorname{tr}_{\Omega} w$, where $w \in H_{0, L}^{s}(\mathcal{C})$ solves

$$
\begin{cases}-\operatorname{div}\left(y^{1-2 s} \nabla w\right)=0 & \text { in } \mathcal{C}, \\ w=0 & \text { on } \partial_{L} \mathcal{C}, \\ \partial_{\nu}^{s} w=g(x) & \text { on } \Omega\end{cases}
$$

As in [16] (see also [7]), the above definition is well defined and

$$
T_{s} \circ(-\Delta)^{s}=\left.i d\right|_{H_{0}^{s}(\Omega)}, \quad(-\Delta)^{s} \circ T_{s}=\left.i d\right|_{H^{-s}(\Omega)},
$$

which implies $T_{s}$ is the inverse of the operator $(-\Delta)^{s}$. Denote $T_{s}$ by $(-\Delta)^{-s}$. Clearly,

$$
(-\Delta)^{-s}=\left((-\Delta)^{s}\right)^{-1}
$$

Moreover, by Proposition 4.2 in [16], the operator $(-\Delta)^{-s}$ is a self-adjoint and positive compact operator.

Now we define the operator $A_{\varepsilon}: H_{0}^{s}(\Omega) \rightarrow H_{0}^{s}(\Omega)$ by

$$
A_{\varepsilon}(u)=(-\Delta)^{-s}\left[\lambda u+|u|^{2_{s}^{*}-2-\varepsilon} u\right]
$$

for $u \in H_{0}^{s}(\Omega)$. Then the gradient of $I_{\varepsilon}$ has the form

Indeed, we have

$$
I_{\varepsilon}^{\prime}(u)=u-A_{\varepsilon}(u) \text {. }
$$

$$
\begin{aligned}
& \left\langle I_{\varepsilon}^{\prime}(u), \varphi\right\rangle_{H^{-s}(\Omega), H_{0}^{s}(\Omega)}=\int_{\Omega}(-\Delta)^{s / 2} u(-\Delta)^{s / 2} \varphi d x-\int_{\Omega}\left(\lambda u+|u|^{2_{s}^{*}-2-\varepsilon} u\right) \varphi d x \\
& =\int_{\Omega}(-\Delta)^{s / 2} u(-\Delta)^{s / 2} \varphi d x-\int_{\Omega}(-\Delta)^{s} A_{\varepsilon}(u) \varphi d x \\
& =\int_{\Omega}(-\Delta)^{s / 2} u(-\Delta)^{s / 2} \varphi d x-\int_{\Omega}(-\Delta)^{s / 2} A_{\varepsilon}(u)(-\Delta)^{s / 2} \varphi d x \\
& =\left\langle u-A_{\varepsilon}(u), \varphi\right\rangle_{H_{0}^{s}(\Omega)}, \quad \forall u, \varphi \in H_{0}^{s}(\Omega) .
\end{aligned}
$$


INFINITELY MANY SIGN-CHANGING SOLUTIONS FOR ... 1155

Note that the set of fixed points of $A_{\varepsilon}$ is the same as the set of critical points of $I_{\varepsilon}$, which is $\mathcal{K}_{\varepsilon}:=\left\{u \in H_{0}^{s}(\Omega): I_{\varepsilon}^{\prime}(u)=0\right\}$.

3.3. Invariant subsets of descending flow. It is easy to check that $I_{\varepsilon}^{\prime}$ is locally Lipschitz continuous. We consider the negative gradient flow $\varphi_{\varepsilon}$ of $I_{\varepsilon}$ defined by

$$
\left\{\begin{array}{l}
\frac{d}{d t} \varphi_{\varepsilon}(t, u)=-I_{\varepsilon}^{\prime}\left(\varphi_{\varepsilon}(t, u)\right) \text { for } t \geq 0, \\
\varphi_{\varepsilon}(0, u)=u .
\end{array}\right.
$$

Here and in the sequel, define the convex cones

$$
P^{+}=\left\{u \in H_{0}^{s}(\Omega): u \geq 0\right\} \quad \text { and } \quad P^{-}=\left\{u \in H_{0}^{s}(\Omega): u \leq 0\right\} .
$$

For $\vartheta>0$, we denote

$$
P_{\vartheta}^{+}=\left\{u \in H_{0}^{s}(\Omega): \operatorname{dist}\left(u, P^{+}\right)<\vartheta\right\}
$$

and

$$
P_{\vartheta}^{-}=\left\{u \in H_{0}^{s}(\Omega): \operatorname{dist}\left(u, P^{-}\right)<\vartheta\right\},
$$

where $\operatorname{dist}\left(u, P^{ \pm}\right)=\inf _{v \in P^{ \pm}}\|u-v\|_{H_{0}^{s}(\Omega)}$. Obviously, $P_{\vartheta}^{-}=-P_{\vartheta}^{+}$. Let

$$
W=P_{\vartheta}^{+} \cup P_{\vartheta}^{-} .
$$

Then, $W$ is an open and symmetric subset of $H_{0}^{s}(\Omega)$ and $Q:=H_{0}^{s}(\Omega) \backslash W$ contains only sign-changing functions. By similar arguments as in [16] (see also [13]) and [19], we have the following result which shows that for $\vartheta$ small, $P_{\vartheta}^{ \pm}$is an invariant set and all sign-changing solutions to (2.3) are contained in $Q$.

Lemma 3.4. There exists $\vartheta_{0}>0$ such that for any $\vartheta \in\left(0, \vartheta_{0}\right]$, there holds

and

$$
A_{\varepsilon}\left(\partial P_{\vartheta}^{ \pm}\right) \subset P_{\vartheta}^{ \pm}
$$

$$
\varphi_{\varepsilon}(t, u) \in P_{\vartheta}^{ \pm} \text {for all } t>0 \text { and } u \in \overline{P_{\vartheta}^{ \pm}} .
$$

Moreover, every nontrivial solutions $u \in P_{\vartheta}^{+}$and $u \in P_{\vartheta}^{-}$of (2.3) are positive and negative, respectively.

REMARK 3.1. Note that there exists a constant $C>0$ independent of $p \in\left[2,2^{*}\right]$ such that $\|u\|_{p} \leq C\|u\|_{2^{*}}$ for all $p \in\left[2,2^{*}\right]$, as in the proof of Lemma 5.2 in [16], one can show that there exists $\vartheta_{0}>0$ such that for any $\vartheta \in\left(0, \vartheta_{0}\right]$, there holds $A_{\varepsilon}\left(\partial P_{\vartheta}^{ \pm}\right) \subset P_{\vartheta}^{ \pm}$for all $\varepsilon>0$ small enough. 
In the following, we may choose an $\vartheta>0$ small enough such that $P_{\vartheta}^{ \pm}$is an invariant set. In order to construct nodal solution by using the combination of invariant sets method and minimax method, we need a deformation lemma in the presence of invariant sets. Since $I_{\varepsilon}$ satisfies the (PS) condition, using similar arguments to Lemma 5.1 in [20], we have the following result.

Lemma 3.5. Define $\mathcal{K}_{\varepsilon, c}^{1}:=\mathcal{K}_{\varepsilon, c} \cap W, \mathcal{K}_{\varepsilon, c}^{2}:=\mathcal{K}_{\varepsilon, c} \cap Q$, where $\mathcal{K}_{\varepsilon, c}:=$ $\left\{u \in H_{0}^{s}(\Omega): I_{\varepsilon}(u)=c, I_{\varepsilon}^{\prime}(u)=0\right\}$. Let $\varrho>0$ be such that $\left(\mathcal{K}_{\varepsilon, c}^{1}\right)_{\varrho} \subset W$ where $\left(\mathcal{K}_{\varepsilon, c}^{1}\right)_{\varrho}:=\left\{u \in H_{0}^{s}(\Omega): \operatorname{dist}\left(u, \mathcal{K}_{\varepsilon, c}^{1}\right)<\varrho\right\}$. Then there exists an $\delta_{0}>0$ such that for any $0<\delta<\delta_{0}$, there exists $\eta \in C([0,1] \times$ $\left.H_{0}^{s}(\Omega), H_{0}^{s}(\Omega)\right)$ satisfying:

(i) $\eta(t, u)=u$ for $t=0$ or $u \notin I_{\varepsilon}^{-1}\left(\left[c-\delta_{0}, c+\delta_{0}\right]\right) \backslash\left(\mathcal{K}_{\varepsilon, c}^{2}\right)_{\varrho}$.

(ii) $\eta\left(1, I_{\varepsilon}^{c+\delta} \cup W \backslash\left(\mathcal{K}_{\varepsilon, c}^{2}\right)_{3 \varrho}\right) \subset I_{\varepsilon}^{c-\delta} \cup W$ and $\eta\left(1, I_{\varepsilon}^{c+\delta} \cup W\right) \subset I_{\varepsilon}^{c-\delta} \cup W$ if $\mathcal{K}_{\varepsilon, c}^{2}=\emptyset$. Here $I_{\varepsilon}^{d}=\left\{u \in H_{0}^{s}(\Omega): I_{\varepsilon}(u) \leq d\right\}$ for any $d \in \mathbb{R}$.

(iii) $\eta(t, \cdot)$ is odd and a homeomorphism of $H_{0}^{s}(\Omega)$ for $t \in[0,1]$.

(iv) $I_{\varepsilon}(\eta(\cdot, u))$ is non-increasing.

(v) $\eta(t, W) \subset W$ for any $t \in[0,1]$.

3.4. Existence of infinitely many sign-changing solutions. Now we prove the existence of infinitely many sign-changing solutions to problem (1.1).

Proof of Theorem 1.1. Here and in the sequel, we fix $\lambda \in\left(0, \lambda_{1}^{s}\right)$. As in [30], we divide the proof into three steps.

Step 1. For any $\varepsilon \in\left(0,2^{*}-\zeta\right)$ small, we define the minimax value $c_{\varepsilon, k}$ for the perturbed functional $I_{\varepsilon}(u)$ with $k=2,3, \cdots$. Set

$$
G_{m}:=\left\{h \in C\left(\mathcal{B}_{R} \cap E_{m}, H_{0}^{s}(\Omega)\right): h \text { is odd and } h=i d \text { on } \partial \mathcal{B}_{R} \cap E_{m}\right\},
$$

where $R>0$ is given by Lemma [3.2. Note that $i d \in G_{m}$, thus $G_{m} \neq \emptyset$. For $k \geq 2$, we define

$\Gamma_{k}:=\left\{h\left(\mathcal{B}_{R} \cap E_{m} \backslash Y\right): h \in G_{m}, m \geq k, Y=-Y\right.$ is open and $\left.\gamma(Y) \leq m-k\right\}$, where $\gamma(K)$ is the Krasnoselskii genus of the symmetric closed se $K$, i.e. the smallest integer $n$ such that there exists and odd continuous map $\sigma$ : $K \rightarrow S^{n-1}$. From [22], $\Gamma_{k}$ possess the following properties:

$\left(1^{\circ}\right) \Gamma_{k} \neq \emptyset$ and $\Gamma_{k+1} \subset \Gamma_{k}$ for all $k \geq 2$.

$\left(2^{\circ}\right)$ If $\phi \in C\left(H_{0}^{s}(\Omega), H_{0}^{s}(\Omega)\right)$ is odd and $\phi=i d$ on $\partial \mathcal{B}_{R} \cap E_{m}$, then $\phi(A) \in \Gamma_{k}$ if $A \in \Gamma_{k}$ for all $k \geq 2$.

$\left(3^{\circ}\right)$ If $A \in \Gamma_{k}, Z=-Z$ is open and $\gamma(Z) \leq s<k$ and $k-s \geq 2$, then $A \backslash Z \in \Gamma_{k-s}$. 
INFINITELY MANY SIGN-CHANGING SOLUTIONS FOR . . 1157

Now, for $k=2,3, \cdots$, we can define the minimax value $c_{\varepsilon, k}$ given by

$$
c_{\varepsilon, k}:=\inf _{A \in \Gamma_{k}} \sup _{u \in A \cap Q} I_{\varepsilon}(u) .
$$

We need to show that $c_{\varepsilon, k}(k \geq 2)$ are well defined (that is for any $A \in \Gamma_{k}$, $A \cap Q \neq \emptyset)$ and $c_{\varepsilon, k} \geq \alpha_{\varepsilon}>0$, where $\alpha_{\varepsilon}$ is given by Lemma 3.3 .

Consider the attracting domain of 0 in $H_{0}^{s}(\Omega)$ :

$$
\mathcal{D}:=\left\{u \in H_{0}^{s}(\Omega): \varphi_{\varepsilon}(t, u) \rightarrow 0, \text { as } t \rightarrow \infty\right\} .
$$

Note that $\mathcal{D}$ is open, since 0 is a local minimum of $I_{\varepsilon}$ and by the continuous dependence of ODE on initial data. Moreover, $\partial \mathcal{D}$ is an invariant set and

In particular, there holds

$$
\overline{P_{\vartheta}^{+}} \cap \overline{P_{\vartheta}^{-}} \subset \mathcal{D} \text {. }
$$

$$
I_{\varepsilon}(u)>0 \text { for every } u \in \overline{P_{\vartheta}^{+}} \cap \overline{P_{\vartheta}^{-}} \backslash\{0\}
$$

by the similar arguments to Lemma 3.4 in [4]. Now we claim that for any $A \in \Gamma_{k}$ with $k \geq 2$, it holds

Set

$$
A \cap Q \cap \partial \mathcal{D} \neq \emptyset
$$

$$
A=h\left(\mathcal{B}_{R} \cap E_{m} \backslash Y\right)
$$

with $\gamma(Y) \leq m-k$ and $k \geq 2$. Define

$$
\mathcal{O}:=\left\{u \in \mathcal{B}_{R} \cap E_{m}: h(u) \in \mathcal{D}\right\}
$$

Obviously, $\mathcal{O}$ is a bounded open symmetric set with $0 \in \mathcal{O}$ and $\overline{\mathcal{O}} \subset$ $\mathcal{B}_{R} \cap E_{m}$. Thus, by the Borsuk-Ulam theorem that $\gamma(\partial \mathcal{O})=m$ and by the continuity of $h, h(\partial \mathcal{O}) \subset \partial \mathcal{D}$. As a consequence,

and therefore

$$
h(\partial \mathcal{O} \backslash Y) \subset A \cap \partial \mathcal{D},
$$

$$
\gamma(A \cap \partial \mathcal{D}) \geq \gamma(h(\partial \mathcal{O} \backslash Y)) \geq \gamma(\partial \mathcal{O} \backslash Y) \geq \gamma(\partial \mathcal{O})-\gamma(Y) \geq k,
$$

by the "monotone, sub-additive and supervariant" property of the genus (cf. Proposition 5.4 in [29]). Since $P_{\vartheta}^{+} \cap P_{\vartheta}^{-} \cap \partial \mathcal{D}=\emptyset$, one has

$$
\gamma(W \cap \partial \mathcal{D}) \leq 1
$$

Thus for $k \geq 2$, we conclude that

$$
\gamma(A \cap Q \cap \partial \mathcal{D}) \geq \gamma(A \cap \partial \mathcal{D})-\gamma(W \cap \partial \mathcal{D}) \geq k-1 \geq 1,
$$

which proves (3.3). Therefore, it follows from (3.3) that $A \cap Q \neq \emptyset$. Moreover, we have

$$
c_{\varepsilon, 2} \geq \alpha_{\varepsilon}>0
$$

because $\partial \mathcal{B}_{\rho_{\varepsilon}} \subset \mathcal{D}$ and $\sup _{A \cap Q} I_{\varepsilon} \geq \inf _{\partial \mathcal{D}} I_{\varepsilon} \geq \inf _{\partial \mathcal{B}_{\rho_{\varepsilon}}} I_{\varepsilon} \geq \alpha_{\varepsilon}>0$ by Lemma 3.3.

Hence, $c_{\varepsilon, k}$ are well defined for all $k \geq 2$ and $0<\alpha_{\varepsilon} \leq c_{\varepsilon, 2} \leq c_{\varepsilon, 3} \leq \cdots$. 
Now, we claim

$$
\mathcal{K}_{\varepsilon, c_{\varepsilon, k}} \cap Q \neq \emptyset,
$$

which implies that there exists a sign-changing critical point $u_{\varepsilon, k}$ such that

$$
I_{\varepsilon}\left(u_{\varepsilon, k}\right)=c_{\varepsilon, k}
$$

and $c_{\varepsilon, k} \rightarrow \infty$, as $k \rightarrow \infty$. It can be done, using deformation Lemma 3.5 following the same arguments as in the proof of Step 1 in [30].

Step 2. We show that for any fixed $k \geq 2,\left\|u_{\varepsilon, k}\right\|_{H_{0}^{s}(\Omega)}$ is uniformly bounded with respect to $\varepsilon$, and then $u_{\varepsilon, k}$ converges strongly to $u_{k}$ in $H_{0}^{s}(\Omega)$ as $\varepsilon \rightarrow 0$.

In fact, using the same $\Gamma_{k}$ above, we can also define the minimax value for the auxiliary functional $I_{*}$ (see (3.1)) by

$$
\beta_{k}:=\inf _{A \in \Gamma_{k}} \sup _{u \in A} I_{*}(u), \quad k=2,3, \cdots .
$$

Here, choosing $R>0$ sufficiently large if necessary, we point out that Lemma 3.2 also holds for $I_{*}$. Then from a $\mathbb{Z}_{2}$ version of the Mountain Pass Theorem (see Theorem 9.12 in [22]), for each $k \geq 2, \beta_{k}>0$ is well defined and

Since

$$
\beta_{k} \rightarrow \infty, \quad \text { as } k \rightarrow \infty
$$

$$
I_{\varepsilon}(u) \leq I_{*}(u)+\frac{|\Omega|}{2_{s}^{*}}
$$

holds for any $\varepsilon \in\left(0,2_{s}^{*}-\zeta\right)$, by the definition of $c_{\varepsilon, k}$ and $\beta_{k}$, we have

$$
c_{\varepsilon, k} \leq \beta_{k}+\frac{|\Omega|}{2_{s}^{*}} .
$$

Therefore, for fixed $k \geq 2, c_{\varepsilon, k}$ is uniformly bounded for $\varepsilon \in\left(0,2_{s}^{*}-\zeta\right)$, i.e., there exists $C=C\left(\beta_{k}, \Omega\right)>0$ independent on $\varepsilon$, such that $c_{\varepsilon, k} \leq C$ uniformly for $\varepsilon$. Since $u_{\varepsilon, k}$ is a nodal solution of (2.3) and $I_{\varepsilon}\left(u_{\varepsilon, k}\right)=c_{\varepsilon, k}$, one concludes that

$$
\frac{\lambda_{1}^{s}-\lambda}{\lambda_{1}^{s}} \int_{\Omega}\left|(-\Delta)^{s / 2} u_{\varepsilon, k}\right|^{2} d x \leq \int_{\Omega}\left|u_{\varepsilon, k}\right|^{2_{s}^{*}-\varepsilon} d x=\frac{2\left(2_{s}^{*}-\varepsilon\right)}{2_{s}^{*}-\varepsilon-2} c_{\varepsilon, k} \leq C,
$$

which implies that $\left\|u_{\varepsilon, k}\right\|_{H_{0}^{s}(\Omega)} \leq C$ uniformly with respect to $\varepsilon$. Denote

$$
w_{\varepsilon, k}=E_{s}\left(u_{\varepsilon, k}\right) \text {. }
$$

Then, $w_{\varepsilon, k}$ is a solution of (2.4) satisfying $\left\|w_{\varepsilon, k}\right\|_{H_{0, L}^{s}(\mathcal{C})} \leq C$ uniformly with respect to $\varepsilon$. So we can apply Theorem 2.1 and obtain a subsequence $\left\{w_{\varepsilon_{n}, k}\right\}_{n \in \mathbb{N}}$, such that

$$
w_{\varepsilon_{n}, k} \rightarrow w_{k} \text { strongly in } H_{0, L}^{s}(\mathcal{C})
$$

for some $w_{k} \in H_{0, L}^{s}(\mathcal{C})$. We set

Then,

$$
u_{k}=\operatorname{tr}_{\Omega} w_{k} .
$$


INFINITELY MANY SIGN-CHANGING SOLUTIONS FOR . . 1159

$$
u_{\varepsilon_{n}, k} \rightarrow u_{k} \text { strongly in } H_{0}^{s}(\Omega)
$$

by the trace inequality and also $c_{\varepsilon_{n}, k} \rightarrow c_{k}$. Thus $u_{k}$ is a solution of (1.1) and $I\left(u_{k}\right)=c_{k}$. Moreover, since $u_{\varepsilon_{n}, k}$ is sign-changing, similar to Step 1, by Lemma 2.1, we can show that $u_{k}$ is still sign-changing.

Step 3. We are in a position to prove that the functional has infinitely many sign-changing critical points. Recalling that $c_{k}$ is non-decreasing with respect to $k$, we distinguish two cases:

Case I: There exist $2 \leq k_{1}<\cdots<k_{i}<\cdots$, satisfying $c_{k_{1}}<\cdots<$ $c_{k_{i}}<\cdots$.

Case II: There is a positive integer $l$ such that $c_{k}=c$ for all $k \geq l$.

Obviously, in Case I, problem (1.1) has infinitely many sign-changing solutions such that $I\left(u_{i}\right)=c_{k_{i}}$, thus we are done. So we assume in the sequel that Case II holds. From now on, we suppose that there exists a $\delta>0$, such that $I(u)$ has no sign-changing critical point $u$ with

$$
I(u) \in[c-\delta, c) \cup(c, c+\delta]
$$

Otherwise, the result follows.

We claim that

$$
\gamma\left(\mathcal{K}_{c}^{2}\right) \geq 2
$$

where $\mathcal{K}_{c}:=\left\{u \in H_{0}^{s}(\Omega): I(u)=c, I^{\prime}(u)=0\right\}$ and $\mathcal{K}_{c}^{2}=\mathcal{K}_{c} \cap Q$. If it is true, $I(u)$ has infinitely many sign-changing critical points and thus we are done. Here we borrow some ideas used in [11]. Arguing by contradiction, suppose that

$$
\gamma\left(\mathcal{K}_{c}^{2}\right)=1
$$

(note that $\mathcal{K}_{c}^{2} \neq \emptyset$ ). Moreover, we assume $\mathcal{K}_{c}^{2}$ contains only finitely many critical points, otherwise the proof is completed. As a consequence, $\mathcal{K}_{c}^{2}$ is compact. Clearly, $0 \notin \mathcal{K}_{c}^{2}$. Thus there exists a open neighborhood $N$ in $H_{0}^{s}(\Omega)$ with $\mathcal{K}_{c}^{2} \subset N$ such that $\gamma(N)=\gamma\left(\mathcal{K}_{c}^{2}\right)$.

Define

$$
U_{\varepsilon}:=I_{\varepsilon}^{-1}([c-\delta, c+\delta]) \backslash N .
$$

Now we claim that for $\varepsilon>0$ small, $I_{\varepsilon}$ has no sign-changing critical point in $U_{\varepsilon}$. Indeed, if not, we suppose that there exist $\varepsilon_{n} \rightarrow 0$ and $u_{n} \in U_{\varepsilon_{n}}$ satisfying $I_{\varepsilon_{n}}^{\prime}\left(u_{n}\right)=0$, with $u_{n}^{ \pm} \neq 0$, and $u_{n} \notin N$. Obviously,

$$
I_{\varepsilon_{n}}\left(u_{n}\right) \in[c-\delta, c+\delta] \text {. }
$$

Then, similar to (3.5), one can obtain that $\left\|u_{n}\right\|_{H_{0}^{s}(\Omega)} \leq C$ uniformly with respect to $n$. Set

$$
w_{n}=E_{s}\left(u_{n}\right) .
$$


By Lemma $2.1 w_{n}$ is a solution of (2.4) satisfying $\left\|w_{n}\right\|_{H_{0, L}^{s}(\mathcal{C})} \leq C$ uniformly with respect to $n$. Therefore, by Theorem 2.1, we obtain, up to a subsequence, that

$$
w_{n} \rightarrow w \text { strongly in } H_{0, L}^{s}(\mathcal{C})
$$

for some $w \in H_{0, L}^{s}(\mathcal{C})$. We set

$$
u=t r_{\Omega} w
$$

Clearly, $u_{n} \rightarrow u$ strongly in $H_{0}^{s}(\Omega)$. Thus

$$
I^{\prime}(u)=0, \quad I(u) \in[c-\delta, c+\delta] \text { and } u \notin \mathcal{K}_{c}^{2} .
$$

But $u$ is still sign-changing, a contradiction.

From the above observation, one can easily show that for any $\varepsilon>0$ small, there exists a constant $\alpha_{\varepsilon}>0$ such that

$$
\left\|I_{\varepsilon}^{\prime}(u)\right\| \geq \alpha_{\varepsilon}, \quad \text { for } u \in I_{\varepsilon}^{-1}([c-\delta, c+\delta]) \backslash(N \cup W) .
$$

Then, as in [11], standard techniques show that for $\varepsilon>0$ small enough, there exists an odd homeomorphism $\eta \in C\left(H_{0}^{s}(\Omega), H_{0}^{s}(\Omega)\right)$ such that $\eta(u)=$ $u$ for $u \in I_{\varepsilon}^{c-2 \delta}$ and

$$
\eta\left(I_{\varepsilon}^{c+\delta} \cup W \backslash N\right) \subset I_{\varepsilon}^{c-\delta} \cup W .
$$

See for example the proof of Theorem A.4 in [22] and also Lemma 5.1 in [20].

Now fix $k>l$. Since $c_{\varepsilon, k}, c_{\varepsilon, k+1} \rightarrow c$ as $\varepsilon \rightarrow 0$, we can find an $\varepsilon>0$ small, such that

$$
c_{\varepsilon, k}, c_{\varepsilon, k+1} \in\left(c-\frac{\delta}{4}, c+\frac{\delta}{4}\right) .
$$

By the definition of $c_{\varepsilon, k+1}$, we can find a set $A \in \Gamma_{k+1}, A=h\left(\mathcal{B}_{R} \cap E_{m} \backslash Y\right)$, where $h \in G_{m}, m \geq k+1, \gamma(Y) \leq m-(k+1)$, such that

$$
I_{\varepsilon}(u) \leq c_{\varepsilon, k+1}+\frac{\delta}{4}<c+\frac{\delta}{2}
$$

for any $u \in A \cap Q$, which implies, $A \subset I_{\varepsilon}^{c+\frac{\delta}{2}} \cup W$. Then by (3.6), we have

$$
\eta(A \backslash N) \subset I_{\varepsilon}^{c-\frac{\delta}{2}} \cup W .
$$

Let $\tilde{Y}=Y \cup h^{-1}(N)$. Then $\tilde{Y}$ is symmetric and open, and

$$
\gamma(\widetilde{Y}) \leq \gamma(Y)+\gamma\left(h^{-1}(N)\right) \leq m-(k+1)+1=m-k .
$$

Therefore one can obtain that $\widehat{A}:=\eta\left(h\left(\mathcal{B}_{R} \cap E_{m} \backslash \tilde{Y}\right)\right) \in \Gamma_{k}$ by $\left(2^{\circ}\right)$ and $\left(3^{\circ}\right)$ above. Consequently, by (3.7),

which contradicts to

$$
c_{\varepsilon, k} \leq \sup _{\widehat{A} \cap Q} I_{\varepsilon} \leq \sup _{\eta(A \backslash N) \cap Q} I_{\varepsilon} \leq c-\frac{\delta}{2},
$$

$$
c_{\varepsilon, k}>c-\frac{\delta}{4}
$$


INFINITELY MANY SIGN-CHANGING SOLUTIONS FOR . . 1161

Hence the proof is completed and the functional $I$ has infinitely many signchanging critical points.

\section{Acknowledgements}

L. Li is supported by Research Fund of National Natural Science Foundation of China (No. 11601046), Chongqing Science and Technology Commission (No. cstc2016jcyjA0310), Chongqing Municipal Education Commission (No. KJ1600603), Chongqing Technology and Business University (No. 2015-56-09, 1552007) and Program for University Innovation Team of Chongqing (No. CXTDX201601026).

J. Sun is supported by Research Fund of National Natural Science Foundation of China (No. 11501280) and Natural Science Foundation of Jiangxi Province (No. 20151BAB211001).

The work of S. Tersian is in the frames of the bilateral research project between Bulgarian and Serbian Academies of Sciences, "Analytical and numerical methods for differential and integral equations and mathematical models of arbitrary (fractional or high) order".

\section{References}

[1] D. Averna, S. Tersian, E. Tornator, On the existence and multiplicity of solutions for Dirichlet's problem for fractional differential equations. Fract. Calc. Appl. Anal. 19, No 1 (2016), 253-266; DOI: 10.1515/fca2016-0014; https://www.degruyter.com/view/j/fca.2016.19.issue-1/ issue-files/fca.2016.19.issue-1.xml.

[2] B. Barrios, E. Colorado, A. de Pablo and U. Sánchez, On some critical problems for the fractional Laplacian operator. J. Differential Equations 252, No 11 (2012), 6133-6162.

[3] P. Bartolo, V. Benci, D. Fortunato, Abstract critical point theorems and applications to some nonlinear problems with strong resonance at infinity. Nonlinear Anal. 7, No 9 (1983), 981-1012.

[4] T. Bartsch, Z. Liu and T. Weth, Sign changing solutions of superlinear Schrödinger equations. Commun. Partial Differential Equations 29, No 1-2 (2004), 25-42.

[5] C. Brändle, E. Colorado, A. de Pablo and U. Sánchez, A concaveconvex elliptic problem involving the fractional Laplacian. Proc. Roy. Soc. Edinburgh Sect. A 143, No 1 (2013), 39-71.

[6] H. Brézis and L. Nirenberg, Positive solutions of nonlinear elliptic equations involving critical Sobolev exponents. Comm. Pure Appl. Math. 36, No 4 (1983), 437-477. 
[7] X. Cabré and J. Tan, Positive solutions of nonlinear problems involving the square root of the Laplacian. Adv. Math. 224, No 5 (2010), 20522093.

[8] L. Caffarelli, J.M. Roquejoffre and Y. Sire, Variational problems for free boundaries for the fractional Laplacian. J. Eur. Math. Soc. 12, No 5 (2010), 1151-1179.

[9] L. Caffarelli, S. Salsa and L. Silvestre, Regularity estimates for the solution and the free boundary of the obstacle problem for the fractional Laplacian. Invent. Math. 171, No 2 (2008), 425-461.

[10] L. Caffaralli and L. Silvestre, An extension problem related to the fractional Laplacian. Comm. Partial Differential Equations 32, No 8 (2007), 1245-1260.

[11] D. Cao, S. Peng and S. Yan, Infinitely many solutions for $p$-Laplacian equation involving critical Sobolev growth. J. Funct. Anal. 262, No 6 (2012), 2861-2902.

[12] D. Cao and S. Yan, Infinitely many solutions for an elliptic problem involving critical Sobolev growth and Hardy potential. Calc. Var. Partial Differential Equations 38, No 3 (2010), 471-501.

[13] A. Capella, Solutions of a pure critical exponent problem involving the half-Laplacian in annular-shaped domains. Comm. Pure Appl. Anal. 10, No 6 (2011), 1645-1662.

[14] G. Cerami, D. Fortunato and M. Struwe, Bifurcation and multiplicity results for nonlinear elliptic problems involving critical Sobolev exponents. Ann. Inst. H. Poincaré Anal. Non Linéarier 1, No 23 (1984), 341-350.

[15] G. Cerami, S. Solimini and M. Struwe, Some existence results for superlinear elliptic boundary value problems involving critical exponents. J. Funct. Anal. 69, No 3 (1986), 289-306.

[16] X. Chang and Z.-Q. Wang, Nodal and multiple solutions of nonlinear problems involving the fractional Laplacian. J. Differential Equaitons 256, No 8 (2014), 2965-2992.

[17] M. Clapp and T. Weth, Multiple solutions for the Brézis-Nirenberg problem. Adv. Differ. Equ. 10, No 4 (2005), 463-480.

[18] G. Devillanova and S. Solimini, Concentration estimates and multiple solutions to elliptic problems at critical growth. Adv. Differ. Equ. 7, No 10 (2002), 1257-1280.

[19] Z. Liu and J. Sun, Invariant sets of descending flow in critical point theory with applications to nonlinear differential equaitons. J. Differential Equations 172, No 2 (2001), 257-299. 
INFINITELY MANY SIGN-CHANGING SOLUTIONS FOR . . 1163

[20] Z. Liu, F. van Heerden and Z.-Q. Wang, Nodal type bound states of Schrödinger equations via invariant set and minimax methods. $J$. Differential Equations 214, No 2 (2005), 358-390.

[21] P. K. Mishra, K. Sreenadh, Bifurcation and multiplicity of solutions for the fractional Laplacian with critical exponential nonlinearity. Electron. J. Differential Equations 2016, No 203 (2016), 1-9.

[22] P.H. Rabinowitz, Minimax Methods in Critical Point Theory with Applications to Differential Equations. CBMS Regional Conference Ser. in Mathematics, Vol. 65, Amer. Math. Soc., Providence, RI (1986).

[23] M. Schechter and W. Zou, On the Brézis-Nirenberg problem. Arch. Rational Mech. Anal. 197, No 1 (2010), 337-356.

[24] R. Servadei, A critical fractional Laplace equation in the resonant case. Topol. Methods Nonlinear Anal. 43, No 1 (2014), 251-267.

[25] R. Servadei and E. Valdinoci, The Brezis-Nirenberg result for the fractional Laplacian. Trans. Amer. Math. Soc. 367, No 1 (2015), 67-102.

[26] R. Servadei and E. Valdinoci, A Brezis-Nirenberg result for non-local critical equations in low dimension. Commun. Pure Appl. Anal. 12, No 6 (2013), 2445-2464.

[27] L. Silvestre, Regularity of the obstacle problem for a fractional power of the Laplace operator. Comm. Pure Appl. Math. 60, No 1 (2006), 67-112.

[28] M. Struwe, A global compactness result for elliptic boundary value problems involving limiting nonlinearities. Math. Z. 187, No 4 (1984), $511-517$.

[29] M. Struwe, Variational Methods. Applications to Nonlinear Partial Differential Equations and Hamiltonian Systems. $3^{\text {rd }}$ Ed., Springer-Verlag, Berlin (2000).

[30] J. Sun and S. Ma, Infinitely many sign-changing solutions for the Brézis-Nirenberg problem. Commun. Pure Appl. Anal. 13, No 6 (2014), 2317-2330.

[31] J. Tan, The Brezis-Nirenberg type problem involving the square root of the Laplacian. Calc. Var. Partial Differential Equations 42, No 1 (2011), 21-41.

[32] J. Xu, D. O'Regan, K. Zhang, Multiple solutions for a class of fractional Hamiltonian systems. Fract. Calc. Appl. Anal. 18, No 1 (2015), 48-63; DOI: 10.1515/fca-2015-0005; https://www.degruyter.com/ view/j/fca.2015.18.issue-1/issue-files/fca.2015.18.issue-1.xml.

[33] S. Yan and J. Yang, Infinitely many solutions for an elliptic problem involving critical Sobolev and Hardy-Sobolev exponents. Calc. Var. Partial Differential Equations 48, No 3-4 (2013), 587-610. 
[34] S. Yan, J. Yang and X. Yu, Equations involving fractional Laplacian operator: Compactness and applications. J. Funct. Anal. 269, No 1 (2015), 47-79.

[35] X. Yu, The Nehari manifold for elliptic equation involving the square root of the Laplacian, J. Differential Equations 252, No 2 (2012), 12831308.

[36] B. Zhang, G. Molica Bisci and R. Servadei, Superlinear nonlocal fractional problems with infinitely many solutions. Nonlinearity $\mathbf{2 8}$, No 7 (2015), 2247-2264.

[37] Q.M. Zhou, K.Q. Wang, Existence and multiplicity of solutions for nonlinear elliptic problems with the fractional Laplacian. Fract. Calc. Appl. Anal. 18, No 1 (2015), 133-145; DOI: 10.1515/fca-2015-0009; https://www.degruyter.com/view/j/fca.2015.18.issue-1/ issue-files/fca.2015.18.issue-1.xml.

${ }^{1}$ School of Mathematics and Statistics

Chongqing Technology and Business University

Chongqing - 400067, CHINA

e-mail: lilin420@gmail.com

2 Department of Mathematics

Nanchang University

Nanchang - 330031, CHINA

e-mail: sunjijiang2005@163.com

Received: February 27, 2017

${ }^{3}$ Department of Mathematics

University of Ruse

Ruse - 7017, BULGARIA

e-mail: sterzian@uni-ruse.bg

${ }^{4}$ Associate at: Institute of Mathematics and Informatics

Bulgarian Academy of Sciences

Sofia - 1113, BULGARIA

Please cite to this paper as published in:

Fract. Calc. Appl. Anal., Vol. 20, No 5 (2017), pp. 1146-1164,

DOI: $10.1515 /$ fca-2017-0061 